\title{
Fontes de magnésia e seu potencial para produção de concretos refratários espinelizados in-situ sem a adição de cimentos refratários
}

\author{
(Magnesia sources and their potential to produce free cement \\ in-situ spinel castables)
}

\author{
T. M. Souza', M. A. L. Braulio' , P. Bonadia ${ }^{2}$ V. C. Pandolfelli ${ }^{I}$ \\ ${ }^{1}$ Grupo de Engenharia de Microestrutura de Materiais - GEMM, Departamento de Engenharia de Materiais \\ Universidade Federal de S. Carlos, Rod. Washington Luiz, km 235, C.P. 676, S. Carlos, SP, 13565-905 \\ ${ }^{2}$ Magnesita Refratários S.A., Centro de Pesquisas e Desenvolvimento, Praça Louis Ensch 240, Contagem, MG \\ uaitiago@gmail.com,vicpando@power.ufscar.br
}

\begin{abstract}
Resumo
Devido à hidratação e seu efeito expansivo, o uso de magnésias reativas em concretos refratários é usualmente evitado. Entretanto, magnésias cáusticas possuem maior área superficial e menor tamanho médio de cristais quando comparadas ao sínter de MgO, podendo favorecer a formação de espinélio $\left(\mathrm{MgAl}_{2} \mathrm{O}_{4}\right)$ em temperaturas menores que as usuais, por aumentar a força motriz para reação da $\mathrm{Al}_{2} \mathrm{O}_{3}$ com o $\mathrm{MgO}$. Além disto, a expansão volumétrica associada à hidratação da magnésia poderia ser utilizada para aumentar a resistência mecânica, viabilizando a substituição do cimento e, conseqüentemente, a redução dos custos destes concretos. Considerando-se estes aspectos, o objetivo do presente trabalho foi avaliar a incorporação de fontes de $\mathrm{MgO}$ com reatividades distintas (um sínter de $\mathrm{MgO}$ e duas magnésias cáusticas) em concretos refratários aluminosos contendo diferentes teores de cimento de aluminato de cálcio $(0,2,4$ ou 6\%-p) e 1\%-p de microssílica. A incorporação do cimento de aluminato de cálcio e da microssílica foi realizada com o intuito de reduzir os danos associados à hidratação do $\mathrm{MgO}$. Como resultado, rotas para incorporação de fontes de magnésias com reatividade distintas aos concretos são apresentadas, sem os danos decorrentes da expansão volumétrica causada pela hidratação, potencializando a redução da temperatura de início de formação de espinélio. Os resultados também indicam que o $\mathrm{MgO}$, para situações particulares, é uma alternativa adequada para a substituição do cimento de aluminato de cálcio como ligante nestes concretos.

Palavras-chave: hidratação da magnésia, cimento de aluminato de cálcio, concretos refratários.
\end{abstract}

\begin{abstract}
Due to its hydration and expansive effect, the use of caustic magnesia in refractory castables is usually not recommended. However, compared to the dead-burned magnesia, the caustic one has a greater surface area and a smaller crystal mean size, which could induce spinel formation $\left(\mathrm{MgAl}_{2} \mathrm{O}_{4}\right)$ at lower temperatures than usual by enhancing the driving force for the reaction between $\mathrm{Al}_{2} \mathrm{O}_{3}$ and $\mathrm{MgO}$. Moreover, the volume expansion associated with the magnesia hydration could be used to develop mechanical strength, allowing the cement replacement and consequently reducing the costs of these castables. Considering these aspects, the objective of the present work was to evaluate the addition of $M g O$ sources with different reactivity (a dead-burned and two caustic magnesias) in refractory castables with various calcium aluminate cement contents (0, 2, 4 or $6 \mathrm{wt} \%)$ and $1 \mathrm{wt} \%$ of fumed silica. The addition of calcium aluminate cement and fumed silica was carried out in order to inhibit the deleterious effects associated to magnesia hydration. As a result, different routes for magnesia sources addition with distinct reactivity to the castables are presented, without the usual damages resulting from the volumetric expansion caused by $\mathrm{MgO}$ hydration, which in parallel will potentially reduce the initial spinel formation temperature. The results also highlighted that magnesia is a suitable alternative to replace calcium aluminate cement as a binder in these castables, for particular compositions.
\end{abstract}

Keywords: magnesia hydration, calcium aluminate cement, refractory castables.

\section{INTRODUÇÃO}

A formação de espinélio de alumina in situ $\left(\mathrm{MgAl}_{2} \mathrm{O}_{4}\right)$ ocorre em temperaturas superiores a $1000{ }^{\circ} \mathrm{C}$ a partir da reação entre a magnésia e a alumina presentes na matriz dos concretos. Esta reação pode ser afetada por diversos parâmetros, como por exemplo: a natureza do agregado utilizado, a quantidade de microssílica presente, o tamanho de partículas de $\mathrm{MgO}$ e sua reatividade, entre outros.
Formulações de concretos espinelizados in situ constituídas por agregados de $\mathrm{Al}_{2} \mathrm{O}_{3}$ favorecem a formação de espinélio mais rico em alumina ( $78 \%-\mathrm{p} \mathrm{Al}_{2} \mathrm{O}_{3}$ e 22\%-p MgO), enquanto agregados de magnésia eletrofundida resultam em espinélio estequiométrico ( $71 \%$ - $\mathrm{p} \mathrm{Al}_{2} \mathrm{O}_{3}$ e 29\%-p MgO), após queima a $1500{ }^{\circ} \mathrm{C}$ [1]. Por sua vez, a adição de microssílica auxilia a acomodação da expansão gerada durante a reação de espinelização e, conseqüentemente, reduz a variação dimensional do produto [2]. Em relação às fontes de magnésia, 
partículas mais grosseiras apresentam menor reatividade, o que retarda sua reação com a alumina para formar o $\mathrm{MgAl}_{2} \mathrm{O}_{4}$ [3]. Por outro lado, magnésias com maior área superficial específica apresentam maiores valores de atividade, favorecendo a formação de uma quantidade superior de espinélio e a redução da temperatura de início de reação entre o $\mathrm{MgO}$ e $\mathrm{Al}_{2} \mathrm{O}_{3}[4,5]$. No entanto, ao entrar em contato com a água durante as etapas de processamento dos concretos, o $\mathrm{MgO}$ hidrata-se facilmente gerando brucita $\left[\mathrm{Mg}(\mathrm{OH})_{2}\right]$. Esta reação é acompanhada por uma expansão volumétrica, devido à diferença de densidade entre a magnésia $\left(\rho_{\mathrm{MgO}}=\right.$ $\left.3,5 \mathrm{~g} / \mathrm{cm}^{3}\right)$ e o hidróxido de magnésio $\left(\rho_{\mathrm{Mg}(\mathrm{OH})_{2}}=2,4 \mathrm{~g} / \mathrm{cm}^{3}\right)$, podendo resultar na formação de trincas e, consequentemente, no comprometimento das propriedades do material. Por isto, o uso de magnésias mais reativas em concretos refratários é sempre evitado. Sendo assim, sua utilização só pode ser viabilizada por meio da adição de cimento e aditivos antihidratação na composição refratária.

A adição de cimento $[6,7]$ e de microssílica [8] são os mecanismos mais efetivos para o controle dos efeitos deletérios associados à hidratação do $\mathrm{MgO}$. No caso da adição de cimento de aluminato de cálcio (CAC), foi recentemente demonstrado para concretos contendo sínter de $\mathrm{MgO}$ que teores baixos (1,5-3\%-p) ou elevados (12\%-p) de CAC resultam em menores danos [7]. Entretanto, aumentandose o teor de CAC observa-se a formação de uma maior quantidade de hexaluminato de cálcio $\left(\mathrm{CA}_{6}\right)$ em temperaturas $>1400{ }^{\circ} \mathrm{C}$, o que resulta em um aumento considerável do volume do corpo [9], uma vez que esta reação também possui caráter expansivo. Sendo assim, o controle da quantidade de cimento fica limitado a teores baixos ou médios e, a fim de alcançar melhores resultados, deve ser aplicado em combinação com um aditivo anti-hidratação. A microssílica, comumente utilizada em concretos refratários, atua reduzindo a velocidade de hidratação da magnésia por meio da formação de um silicato de magnésio hidratado na superfície das partículas de $\mathrm{MgO}[8,10]$. Sua adição nos concretos também proporciona uma melhora significativa no empacotamento das partículas, devido ao efeito lubrificante decorrente de sua morfologia esférica e seu pequeno tamanho de partícula $(<1 \mu \mathrm{m})$ que se acomoda nos interstícios disponíveis, contribuindo para a redução da porosidade [11]. Entretanto, quando o cimento de aluminato de cálcio e microssílica estão presentes na matriz, fases de baixo ponto de fusão são formadas durante o uso, tais como anortita $\left(\mathrm{CaAl}_{2} \mathrm{Si}_{2} \mathrm{O}_{8}\right)$ e guelenita $\left(\mathrm{Ca}_{2} \mathrm{Al}_{2} \mathrm{SiO}_{7}\right)$, por meio da reação entre o $\mathrm{CaO}$, $\mathrm{Al}_{2} \mathrm{O}_{3}$ e $\mathrm{SiO}_{2}$, comprometendo as propriedades mecânicas a quente dos concretos [12]. Desta forma, para obter concretos com bom desempenho utilizando CAC (efeito anti-dano) e microssílica (efeito anti-hidratação) de forma combinada, a adição de microssílica deve ser limitada a teores baixos. Considerando-se estes aspectos, o objetivo do presente trabalho foi avaliar a incorporação de fontes de magnésias com reatividades distintas (sínter de $\mathrm{MgO}$ ou magnésias cáusticas) em concretos refratários aluminosos, adicionandose diferentes teores de cimento de aluminato de cálcio $(0,2$, 4 ou 6\%-p) e uma quantidade fixa de microssílica (1\%-p). A combinação do efeito anti-dano do cimento de aluminato de cálcio e anti-hidratação da microssílica foi avaliada durante a cura e a secagem dos concretos refratários, resultando em rotas para a incorporação de fontes de magnésias com diferentes reatividades nestes materiais.

\section{MATERIAIS E MÉTODOS}

Foram produzidas composições de concreto refratário vibrado de alta alumina, contendo 6\%-p de magnésia, um sínter de $\mathrm{MgO}(\mathrm{SM})$ e duas fontes de magnésia cáustica (MC1 e MC2), com respectivas áreas superficiais iguais a $1,05 \mathrm{~m}^{2} / \mathrm{g}, 11,12 \mathrm{~m}^{2} / \mathrm{g}$ e $24,57 \mathrm{~m}^{2} / \mathrm{g}$ (Magnesita Refratários S.A., Brasil), cujas propriedades físicas e composições químicas são apresentadas em detalhes na Tabela I, 7\%-p de alumina reativa (CL370, Almatis, EUA), 1\%-p de microssílica (971U, Elkem, Noruega), e teores distintos de cimento de aluminato de cálcio - 0 a 6\%-p (Secar 71, Kerneos, França). Formulações sem magnésia e contendo 2, 4 ou 6\%-p de cimento aluminoso foram preparadas para comparação com os concretos contendo $\mathrm{MgO}$. Aluminas tabulares ( $\mathrm{d} \leq 6 \mathrm{~mm}$, Almatis, EUA) foram utilizadas como agregados e a dispersão foi garantida adicionandose $0,2 \%$-p de um aditivo dispersante eletroestérico (Basf, Alemanha). A formulação foi planejada considerando-se o modelo de empacotamento de Alfred $(\mathrm{q}=0,26)$ e o teor de água para cada composição foi ajustado de acordo com um alvo de fluidez inicial sob vibração de $80 \%$, visando-se uma moldagem adequada. A Tabela II apresenta as principais características das composições avaliadas e a Tabela III o teor de água utilizado para o processamento dos concretos.

Os concretos foram moldados e em seguida curados a $50{ }^{\circ} \mathrm{C}$ em uma câmara climática (Vöetsch 2020, Alemanha)

Tabela I - Propriedades físicas e composição química das fontes de magnésia selecionadas.

[Table I - Physical properties and chemical composition of the selected magnesia sources.]

\begin{tabular}{cllll}
\hline Fonte de $\mathrm{MgO}$ & & $\mathrm{SM}$ & $\mathrm{MC} 1$ & $\mathrm{MC} 2$ \\
\hline & $\mathrm{AS}\left(\mathrm{m}^{2} / \mathrm{g}\right)$ & 1,05 & 11,12 & 24,57 \\
& $\mathrm{D}_{10}(\mu \mathrm{m})$ & 0,55 & 2,05 & 2,00 \\
Propriedades & $\mathrm{D}_{50}(\mu \mathrm{m})$ & 7,76 & 20,14 & 16,58 \\
físicas & $\mathrm{D}_{90}(\mu \mathrm{m})$ & 35,48 & 52,19 & 51,09 \\
& $\rho\left(\mathrm{g} / \mathrm{cm}^{3}\right)$ & 3,53 & 3,20 & 3,38 \\
\hline & $\mathrm{MgO}$ & 98,17 & 94,73 & 98,38 \\
& $\mathrm{CaO}$ & 0,84 & 0,42 & 0,88 \\
Composição & $\mathrm{SiO}_{2}$ & 0,33 & 1,58 & 0,17 \\
química & $\mathrm{Al}_{2} \mathrm{O}_{3}$ & 0,12 & 0,35 & 0,05 \\
(\%-p) & $\mathrm{Fe}_{2} \mathrm{O}_{3}$ & 0,41 & 2,06 & 0,42 \\
& $\mathrm{MnO}$ & 0,13 & 0,86 & 0,10 \\
& $\mathrm{CaO} / \mathrm{SiO}_{2}$ & 2,54 & 0,27 & 5,18 \\
\hline
\end{tabular}

$A S$ : área superficial; $D_{90}, D_{50}$ e $D_{10}$ : diâmetro esférico equivalente abaixo do qual se encontram $90 \%, 50 \%$ e $10 \%$ em peso das particulas; $\rho$ : densidade. 
Tabela II - Composições dos concretos refratários estudados.

[Table II - Compositions of the evaluated refractory castables.]

\begin{tabular}{lccccccc}
\hline \multirow{2}{*}{ Matérias primas } & \multicolumn{4}{c}{ Contendo $\mathrm{MgO}$ (SM ou MC1 ou MC2) } & \multicolumn{3}{c}{ Sem MgO } \\
& 0CAC & 2CAC & 4CAC & 6CAC & 2CAC & 4CAC & 6CAC \\
\hline Alumina tabular $(\mathrm{d} \leq 6 \mathrm{~mm})$ & 80 & 80 & 80 & 80 & 80 & 80 & 80 \\
Alumina reativa (CL370) & 7 & 11 & 9 & 7 & 11 & 9 & 7 \\
Microssílica (971 U) & 1 & 1 & 1 & 1 & 1 & 1 & 1 \\
Magnésia (SM ou MC1 ou MC2) & 6 & 6 & 6 & 6 & 0 & 0 & 0 \\
Cimento de aluminato de cálcio (Secar 71) & 0 & 2 & 4 & 6 & 2 & 4 & 6 \\
Alumina tabular $(\mathrm{d}<45 \mu \mathrm{m})$ & 6 & 0 & 0 & 0 & 6 & 6 & 6 \\
Dispersante & 0,2 & 0,2 & 0,2 & 0,2 & 0,2 & 0,2 & 0,2 \\
\hline
\end{tabular}

Tabela III - Teor de água adicionado aos concretos refratários estudados.

[Table III - Water content added to the refractory castables.]

\begin{tabular}{lcccc}
\hline \multirow{2}{*}{ Composição } & \multicolumn{4}{c}{ Teor de água (\%-p) } \\
& 0CAC & 2CAC & 4CAC & 6CAC \\
\hline $\mathrm{SM}$ & 4,1 & 4,0 & 4,1 & 4,2 \\
$\mathrm{MC} 1$ & 5,2 & 4,8 & 5,0 & 5,3 \\
$\mathrm{MC} 2$ & 5,8 & 5,1 & 5,5 & 5,9 \\
Sem $\mathrm{MgO}$ & - & 4,0 & 4,1 & 4,2 \\
\hline
\end{tabular}

com umidade relativa de $80 \%$. O processo de cura foi acompanhado ao longo de 7 dias utilizando-se medidas do módulo de Young, porosidade aparente e resistência mecânica. A determinação do perfil de secagem foi realizada por meio do ensaio de termogravimetria, conforme procedimento mencionado a seguir.

Para avaliação do módulo de Young, os concretos foram moldados na forma de barras retangulares com dimensões aproximadas de $150 \times 25 \times 25 \mathrm{~mm}^{3}$. Após $3 \mathrm{~h}$ de cura, os corpos-de-prova foram cuidadosamente desmoldados e as medidas do módulo elástico foram iniciadas. O módulo de Young foi avaliado por meio da técnica de ressonância de barras utilizando-se o equipamento Scanelastic (ATCP, Brasil), de acordo com a norma ASTM C 1198-91. O arranjo experimental deste ensaio consiste na excitação da amostra e na detecção de seu espectro de vibração por meio da utilização de transdutores piezoelétricos. A excitação é realizada em uma faixa de freqüências suficiente para obtenção das freqüências naturais de ressonância das barras, e, a partir dos modos normais de vibração calcula-se o módulo de Young, utilizando-se as equações de Pickett, que relacionam o módulo elástico, as freqüências naturais de vibração e as dimensões da amostra [13]. Para freqüência fundamental de flexão das barras o módulo de Young é dado por:

$$
\mathrm{E}=0,9465 \frac{\mathrm{mg}_{\mathrm{f}}^{2}}{\mathrm{~b}} \times \frac{\mathrm{L}^{3}}{\mathrm{t}^{3}} \times \mathrm{T}_{1}
$$

na qual E é o módulo de Young (Pa), m a massa (g), b a largura (mm), L o comprimento (mm), t a espessura ( $\mathrm{mm}$ ), $\mathrm{f}_{\mathrm{f}}$ a freqüência fundamental de ressonância da barra em flexão $(\mathrm{Hz})$ e $\mathrm{T}_{1}$ o fator de correção para o modo de flexão fundamental, que considera a espessura finita da barra e a razão de Poisson.

A porosidade aparente (PA) das amostras foi avaliada utilizando-se o método de Arquimedes e querosene como fluido de imersão, de acordo com a norma ASTM C 830. O cálculo é realizado a partir dos valores do peso seco $\left(\mathrm{P}_{\mathrm{s}}\right)$, peso imerso $\left(\mathrm{P}_{\mathrm{i}}\right)$ e peso úmido $\left(\mathrm{P}_{\mathrm{u}}\right)$, dados em gramas, como indicado a seguir:

$$
\mathrm{PA}(\%)=100 \times\left(\frac{\mathrm{P}_{u}-\mathrm{P}_{\mathrm{s}}}{\mathrm{P}_{\mathrm{u}}-\mathrm{P}_{\mathrm{i}}}\right)
$$

A resistência mecânica durante a cura foi medida por compressão diametral segundo a norma ASTM C 496-90, utilizando-se um equipamento MTS (modelo 810, EUA) sob taxa de aplicação de carga constante e igual a $40 \mathrm{~N} / \mathrm{s}$. A tensão de ruptura foi calculada a partir da equação abaixo:

$$
\sigma_{\mathrm{R}}=2 \times\left(\frac{\mathrm{P}_{\max }}{\pi \mathrm{LD}}\right)
$$

na qual $\sigma_{R}$ é a tensão de ruptura (MPa), $\mathrm{P}_{\max }$ a força de ruptura $(\mathrm{N}), \mathrm{L}$ a altura $(\mathrm{m})$ e $\mathrm{D}$ o diâmetro da amostra $(\mathrm{m})$.

Para os ensaios de termogravimetria as amostras foram previamente curadas a $50{ }^{\circ} \mathrm{C}$ por $24 \mathrm{~h}$ e secas por $24 \mathrm{~h}$ a $110{ }^{\circ} \mathrm{C}$ para remoção da água livre, permitindo a avaliação da decomposição dos hidratos associados ao cimento ou à magnésia. Os testes foram conduzidos até $800{ }^{\circ} \mathrm{C}$ em um equipamento de análise termogravimétrica [14], com taxa de aquecimento $10{ }^{\circ} \mathrm{C} / \mathrm{min}$. A perda de massa cumulativa $\mathrm{W}$ e a velocidade de secagem $\mathrm{dW} / \mathrm{dt}$ foram calculadas conforme as equações abaixo:

$$
\mathrm{W}(\%)=100 \times\left(\frac{\mathrm{M}_{0}-\mathrm{M}}{\mathrm{M}_{0}-\mathrm{M}_{\mathrm{f}}}\right)
$$

$$
\left(\frac{\mathrm{dW}}{\mathrm{dt}}\right)_{\mathrm{i}}=\left(\frac{\mathrm{W}_{(\mathrm{i}+10)}-\mathrm{W}_{(\mathrm{i}-10)}}{\mathrm{t}_{(\mathrm{i}+10)}-\mathrm{t}_{(\mathrm{i}-10)}}\right)
$$


as quais $\mathrm{M}_{0}$ é a massa inicial (g), $\mathrm{M}$ a massa instantânea (g) registrada em um tempo $t_{i}(\mathrm{~s})$ e $\mathrm{M}_{\mathrm{f}}$ a massa final da amostra (g). A taxa de secagem é expressa em $\% /$ min.

\section{RESULTADOS E DISCUSSÃO}

\section{Testes de secagem}

Em um trabalho recente dos autores foi apresentada uma avaliação das fontes de magnésia utilizadas (SM, $\mathrm{MC} 1$ e MC2) [15]. Os resultados de termogravimetria das composições $\mathrm{MgO} / \mathrm{H}_{2} \mathrm{O}$ e do tempo de neutralização da magnésia em ácido acético indicaram que quanto maior é a área superficial da fonte de magnésia, maior é o valor da atividade e da perda de massa durante o aquecimento, sendo que a decomposição do $\mathrm{Mg}(\mathrm{OH})_{2}$ formado ocorre na mesma faixa de temperatura (acima de $350{ }^{\circ} \mathrm{C}$ ), independente da fonte de $\mathrm{MgO}$. Com base nas diferenças de perda de massa e nos valores de atividade observados, as fontes de magnésia $\mathrm{SM}, \mathrm{MC} 1$ e MC2 foram classificadas como de reatividade baixa, médio-alta e alta, respectivamente.

Na Fig. 1 são apresentados os resultados de termogravimetria após 1 dia de cura a $50^{\circ} \mathrm{C}$ e secagem por $24 \mathrm{~h} \mathrm{a}$ $110^{\circ} \mathrm{C}$ para os concretos avaliados. Durante a secagem dos concretos refratários, inicialmente ocorre a evaporação da água fisicamente adsorvida e com o aumento da temperatura (acima de $100{ }^{\circ} \mathrm{C}$ ) a água livre passa a ser removida da estrutura por meio da ebulição. No caso de concretos contendo $\mathrm{MgO}$ e/ou CAC, em temperaturas superiores a $100{ }^{\circ} \mathrm{C}$ também há a decomposição dos hidratos do cimento aluminoso e do hidróxido de magnésio presentes. A decomposição destas fases é observada na curva da taxa de secagem por meio de dois picos: o primeiro, abaixo de $400{ }^{\circ} \mathrm{C}$, relacionado à decomposição dos compostos hidratados do CAC $[14,16,17]$ e o segundo, acima de $350^{\circ} \mathrm{C}$, devido à decomposição do hidróxido de magnésio $[16,17]$. O pico associado à decomposição do cimento com água quimicamente ligada pode ser atribuído aos hidratos $\mathrm{C}_{2} \mathrm{AH}_{8}\left(2 \mathrm{CaO} \cdot \mathrm{Al}_{2} \mathrm{O}_{3} \cdot 8 \mathrm{H}_{2} \mathrm{O}\right), \mathrm{AH}_{3}\left(\mathrm{Al}_{2} \mathrm{O}_{3} \cdot 3 \mathrm{H}_{2} \mathrm{O}\right)$ e $\mathrm{C}_{3} \mathrm{AH}_{6}$ ( $\left.3 \mathrm{CaO} \cdot \mathrm{Al}_{2} \mathrm{O}_{3} \cdot 6 \mathrm{H}_{2} \mathrm{O}\right)$, normalmente formados quando a cura é realizada acima de $35{ }^{\circ} \mathrm{C}$, e que se decompõem na faixa de $180-360{ }^{\circ} \mathrm{C}$ [12]. Além disto, deslocamentos nestes intervalos de temperatura podem ocorrer devido ao volume do corpo testado, da sua permeabilidade e da taxa de aquecimento usada (neste estudo $10{ }^{\circ} \mathrm{C} / \mathrm{min}$ ). Como indicado na Fig. 1a para os concretos sem $\mathrm{MgO}$, reduzindo-se o teor de cimento ocorre uma diminuição da quantidade de hidratos de $\mathrm{CAC}$, pois a área abaixo dos picos é
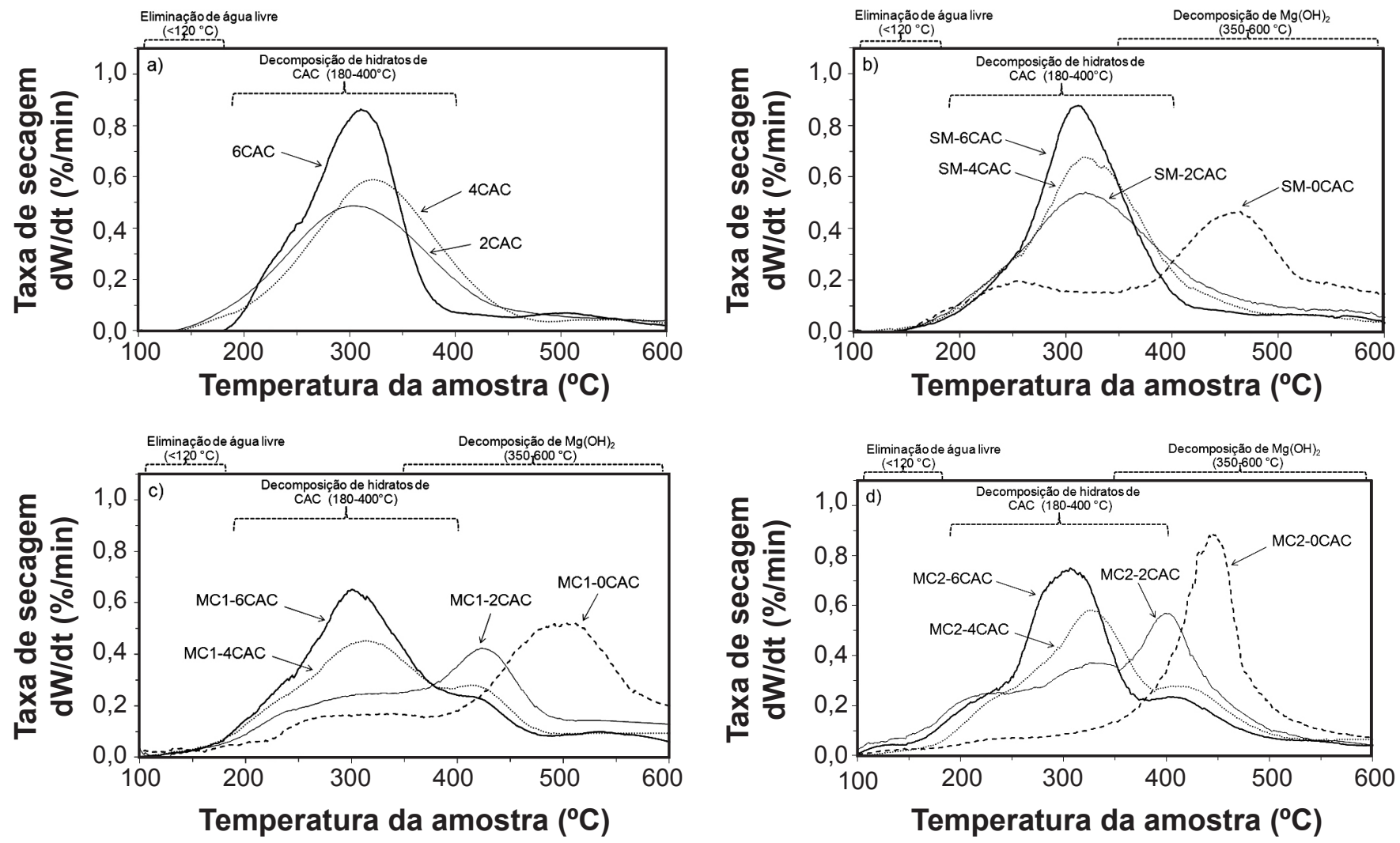

Figura 1: Taxa de secagem em função da temperatura da amostra para concretos contendo diferentes teores de cimento (6CAC, 4CAC, $2 \mathrm{CAC}$ ou $0 \mathrm{CAC}$ ), com ou sem $\mathrm{MgO}$ após 1 dia de cura a $50^{\circ} \mathrm{C}$ e 1 dia de secagem a $110^{\circ} \mathrm{C}$ : a) sem $\mathrm{MgO}$; b) contendo $\mathrm{SM}$; c) contendo $\mathrm{MC} 1$ e d) contendo MC2.

[Figure 1: Drying rate profiles as a function of sample's temperature for castables with different cement contents (6CAC, 4CAC, 2CAC or $O C A C$ ) with or without $\mathrm{MgO}$ after 1 day of curing at $50^{\circ} \mathrm{C}$ and 1 day of drying at $110^{\circ} \mathrm{C}$ : a) $\mathrm{MgO}$-free; b) containing SM; c) containing $M C 1$ and d) containing MC2.] 
proporcional a quantidade do constituinte que se decompõe. Um comportamento semelhante em relação ao teor de CAC também é observado para os concretos contendo fontes distintas de $\mathrm{MgO}$ (Fig. 1b-d), como indicam os picos entre $180{ }^{\circ} \mathrm{C}$ e $400{ }^{\circ} \mathrm{C}$.

Quando a formação de brucita nos concretos estudados é analisada, dois aspectos importantes devem ser considerados: o efeito do cimento de aluminato de cálcio e da microssílica. De acordo com a literatura, a dissolução dos aluminatos de cálcio, durante a reação de hidratação do CAC, resulta em um aumento significativo do $\mathrm{pH}$ e da concentração de íons $\mathrm{OH}^{-}$no meio [19]. Conseqüentemente, devido ao princípio de deslocamento químico, a hidratação do sínter de $\mathrm{MgO}$ é favorecida, enquanto a magnésia cáustica não é muito afetada, uma vez que esta fonte de $\mathrm{MgO}$ é por si só muito mais reativa e sua hidratação também causa um grande aumento do $\mathrm{pH}$ [6]. Por outro lado, para todas as fontes de magnésia, a presença de microssílica resulta na formação de uma camada protetora insolúvel de silicato de magnésio hidratado na superfície das partículas de $\mathrm{MgO}$, reduzindo-se assim sua velocidade de hidratação [8].

Para os concretos contendo CAC (2, 4 ou 6\%-p) e sínter de $\mathrm{MgO}$ (Fig. 1b), o cimento e a microssílica atuam sobre a magnésia simultaneamente, mas os resultados de termogravimetria indicam que o efeito da microssílica (1\%-p, Tabela II) reduzindo a velocidade de hidratação é mais pronunciado, pois a formação de brucita não é claramente observada (não se verifica nenhum pico evidente de decomposição em temperaturas superiores a $350{ }^{\circ} \mathrm{C}$ ). Isto ocorre porque o aumento do $\mathrm{pH}$ associado à hidratação do CAC também favorece a dissolução da microssílica [8] e, conseqüentemente, melhora sua eficiência como aditivo anti-hidratação. Entretanto, quando as magnésias cáusticas são empregadas (Fig. 1c e d), a decomposição da brucita passa a ser observada nos concretos ligados por cimento, pois a quantidade de microssílica adequada é dependente da área superficial da fonte de magnésia [15].

Devido à competição entre o $\mathrm{CAC}$ e o $\mathrm{MgO}$ pela água disponível no meio, à medida que o teor de cimento é reduzido uma quantidade maior de água estará disponível para reagir com a magnésia, podendo resultar em um aumento da hidratação. No caso dos concretos contendo sínter de $\mathrm{MgO}$, observa-se a formação de uma quantidade significativa de brucita apenas para a composição SMOCAC (Fig. 1b). No entanto, para os concretos contendo $\mathrm{MC} 1$ e MC2 nota-se claramente um aumento na intensidade do pico de decomposição da brucita à medida que o teor de CAC é reduzido de 6\%-p para 0\%-p (Fig. 1c e d).

\section{Avaliação da etapa de cura}

Com o aumento do teor de cimento ocorre uma melhoria das propriedades mecânicas dos concretos, pois durante sua hidratação há a precipitação e o crescimento de cristais de hidratos responsáveis pela formação de uma rede ligando as partículas. $\mathrm{O}$ aumento da resistência mecânica resultante da adição de cimento permite que os concretos suportem maiores tensões de tração, devido a expansão associada à hidratação do $\mathrm{MgO}$, sem que ocorram danos. Por outro lado, a formação de hidratos de CAC também é acompanhada por uma redução da porosidade, que diminui a possibilidade de acomodação adequada do hidróxido de magnésio formado [16]. Sendo assim, o efeito do teor de cimento para o concreto contendo $\mathrm{MgO}$ pode ser significativamente alterado quando magnésias mais reativas são utilizadas. Este comportamento foi avaliado para as diferentes fontes de $\mathrm{MgO}$ utilizando-se medidas de módulo de elástico e de porosidade aparente cujos resultados são apresentados nas Figs. 2 e 3, respectivamente.

Para os concretos contendo cimento aluminoso observase no período de cura um aumento gradativo do módulo elástico devido à precipitação dos hidratos [18]. Como conseqüência, reduzindo-se o teor de cimento ocorre uma diminuição do valor do MOE após 7 dias de cura (Fig. 2a). Simultaneamente, para as condições de cura adotadas (50 ${ }^{\circ} \mathrm{C}$ em ambiente com $80 \%$ de umidade relativa), verifica-se para a maioria das composições um aumento da porosidade entre o primeiro e terceiro dia de cura devido à perda de parte da água livre por meio da secagem $[15,18]$. Este comportamento pode ser observado para os concretos $4 \mathrm{CAC}$ e 2 CAC (Fig. 3a). Após este período, pequenas alterações são observadas na porosidade, que permanece praticamente constante para todos os teores de CAC avaliados (Fig. 3a).

Quando o sínter de $\mathrm{MgO}$ é adicionado aos concretos (Fig. 2b), não são observadas diferenças significativas em relação aos concretos sem magnésia, pois a presença de microssílica reduz a quantidade de brucita formada e, conseqüentemente, seu efeito no MOE. No entanto, a expansão volumétrica associada à hidratação da magnésia, quando devidamente acomodada na estrutura dos concretos, reduz o aumento da porosidade e a mobilidade das partículas contribuindo para o aumento da rigidez, como observado para a composição sem cimento (SM-0CAC), onde a magnésia atua como um ligante $[15,18]$. À medida que o teor de CAC é aumentado, de $2 \%$-p para $4 \%$-p ou $6 \%$-p, observa-se um aumento nos valores de MOE (Fig. 2b) e uma redução da porosidade dos concretos (Fig. 3b) devido à formação de uma maior quantidade de hidratos de cimento. Por outro lado, o concreto SM-0CAC apresenta os menores valores de módulo elástico e de porosidade (Figs. 2 b e $3 b$ ), o que está associado principalmente às mudanças no empacotamento de partículas, com a substituição do cimento por alumina tabular $\mathrm{d}<45 \mu \mathrm{m}$ (Tabela II), e às diferenças entre o efeito ligante do cimento e da magnésia.

No caso da adição da fonte de magnésia com reatividade média-alta (MC1), observa-se para os concretos contendo CAC uma aproximação entre os valores de MOE e porosidade após 7 dias de cura (Figs. 2c e 3c) em relação as composições contendo sínter de $\mathrm{MgO}$ (Figs. 2 b e $3 b)$. A variação do teor de CAC (6\%-p a $0 \%$-p) é acompanhada por um aumento na hidratação da magnésia devido à maior disponibilidade de água no meio (Fig. 1c), conseqüentemente, parte da redução da rigidez associada à diminuição do teor de cimento é compensada pelo aumento 

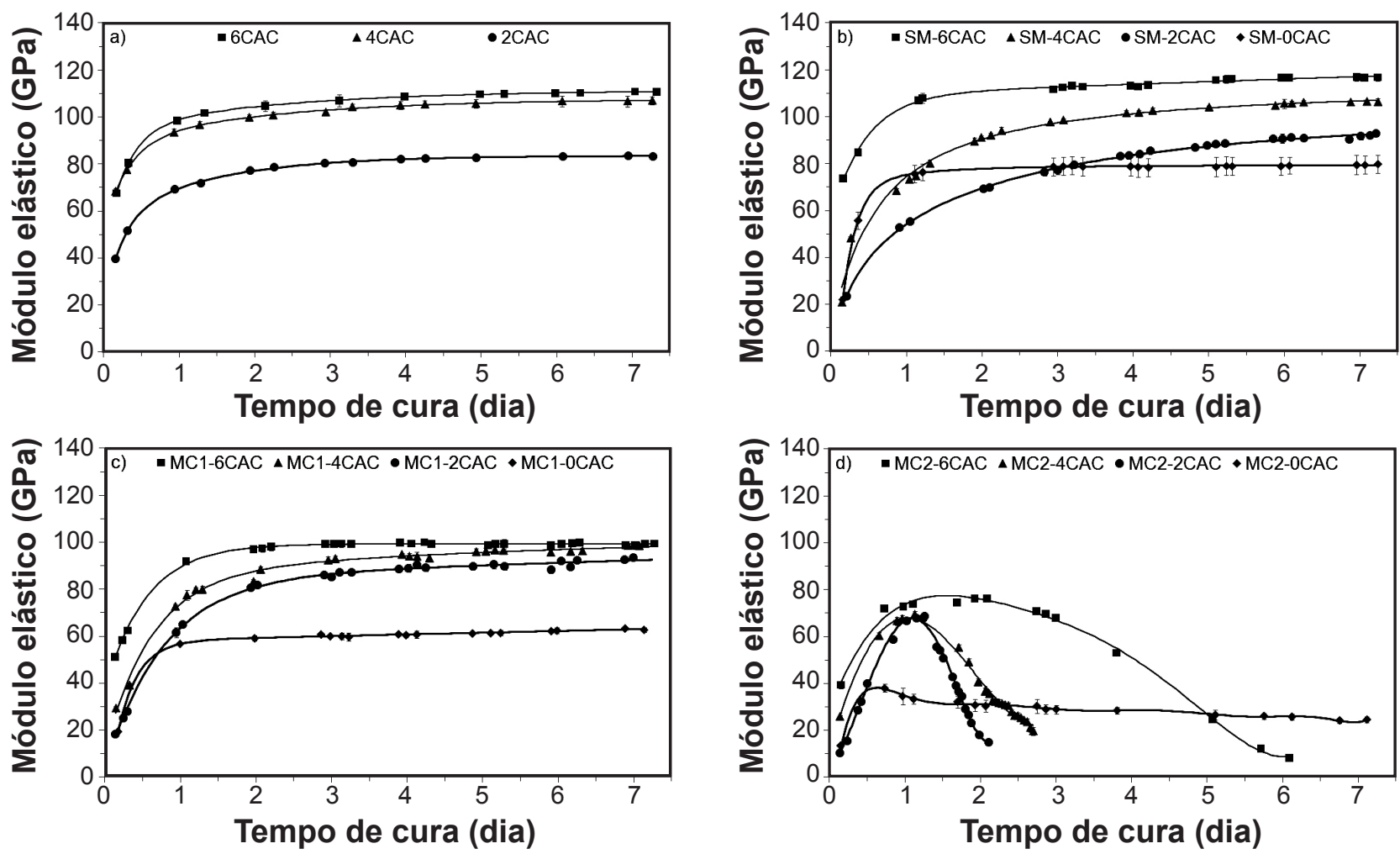

Figura 2: Módulo elástico em função do tempo de cura para concretos contendo diferentes teores de cimento (6CAC, 4CAC, 2CAC ou 0CAC), com ou sem MgO: a) sem $\mathrm{MgO}$; b) contendo $\mathrm{SM}$; c) contendo $\mathrm{MC} 1$ e d) contendo MC2.

[Figure 2: Elastic modulus as a function of curing time for castables with different cement contents (6CAC, 4CAC, 2CAC or 0CAC) with or without $\mathrm{MgO}$ : a) MgO-free; b) containing SM; c) containing MC1 and d) containing MC2.]
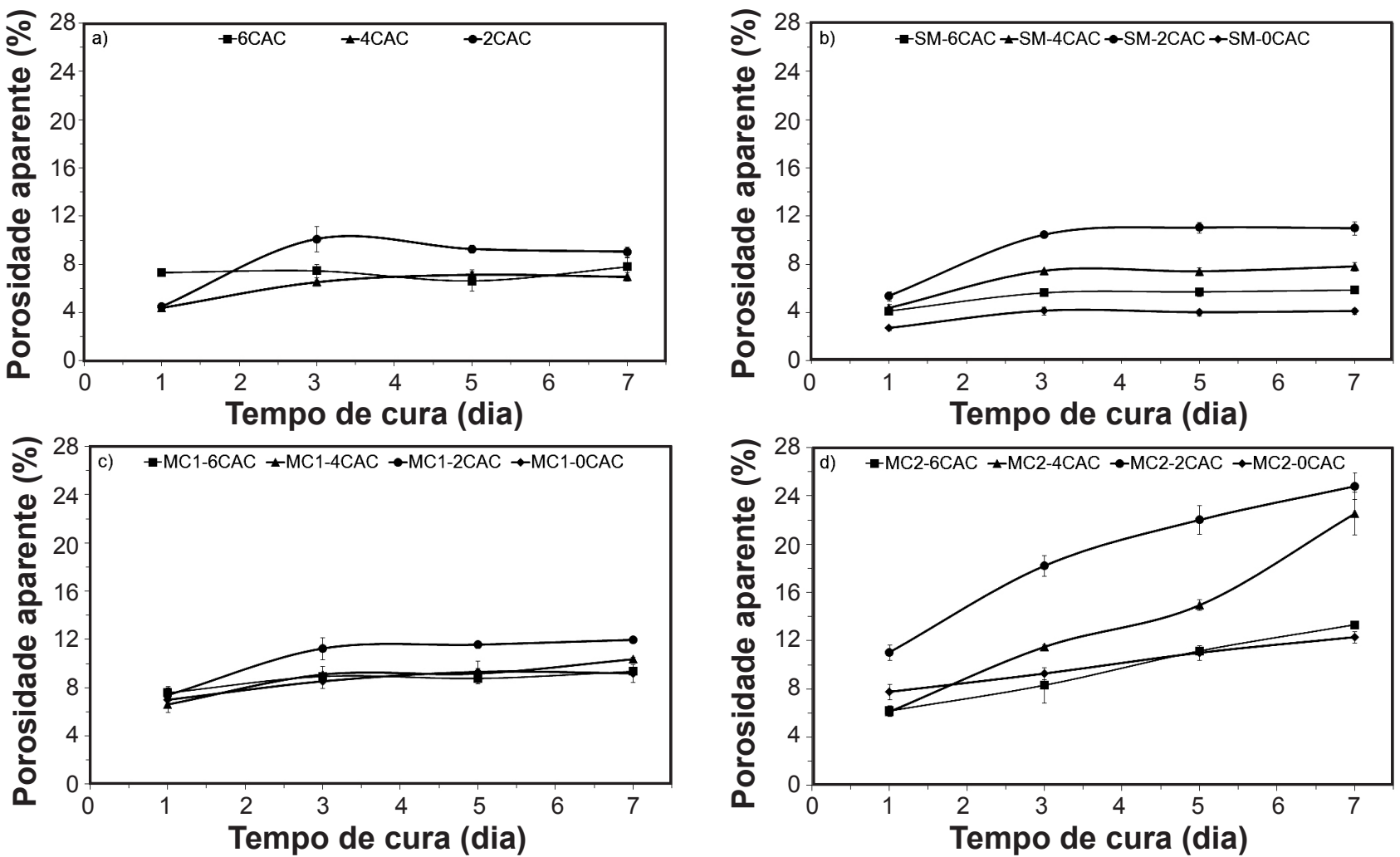

Figura 3: Porosidade aparente em função do tempo de cura para concretos contendo diferentes teores de cimento (6CAC, 4CAC, 2CAC ou 0CAC), com ou sem MgO: a) sem $\mathrm{MgO}$; b) contendo $\mathrm{SM}$; c) contendo $\mathrm{MC1}$ e d) contendo MC2.

[Figure 3: Apparent porosity as a function of curing time for castables with different cement contents (6CAC, 4CAC, 2CAC or 0CAC) with or without $\mathrm{MgO}$ : a) $\mathrm{MgO}$-free; b) containing $\mathrm{SM}$; c) containing $\mathrm{MCl}$ and d) containing $M C 2$. 


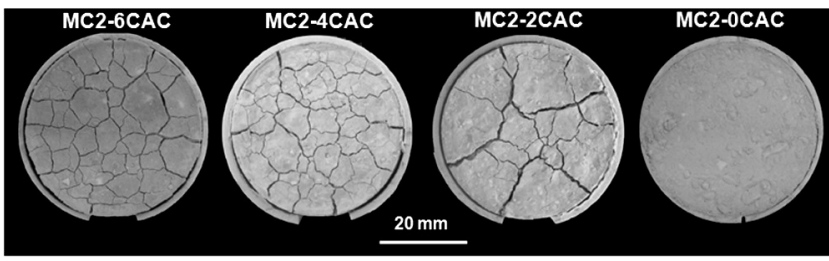

Figura 4: Aspecto visual dos concretos contendo MC2 e diferentes teores de cimento (6CAC, 4CAC, 2CAC e 0CAC) após 7 dias de cura a $50{ }^{\circ} \mathrm{C}$ em ambiente saturado.

[Figure 4: Visual aspects of MC2-containing castables with different cement contents (6CAC, 4CAC, 2CAC e 0CAC) after 7 days of curing at $50{ }^{\circ} \mathrm{C}$ in humid environment.]

do MOE devido à formação de uma quantidade adicional de brucita. Por outro lado, os valores do MOE observados para as composições contendo $\mathrm{MC} 1$ são menores que dos concretos contendo SM (Figs. 2b e 2c), mesmo quando as composições sem cimento são comparadas (SM-0CAC e MC1-0CAC). Este comportamento é resultado do aumento do teor de água utilizado no processamento (Tabela III) devido à maior área superficial das fontes de $\mathrm{MgO}$ mais reativas, que também é responsável pelo aumento da porosidade destes materiais (Figs. 3 b e 3c).

Se uma magnésia ainda mais reativa é incorporada aos concretos (MC2), a expansão associada à sua hidratação resulta numa redução do módulo elástico após algum tempo de cura e num aumento contínuo da porosidade devido à nucleação $\mathrm{e}$ a propagação de trincas (Figs. $2 \mathrm{~d}$ e $3 \mathrm{~d}$ ). Os valores do MOE aumentam no período inicial, atingem a máxima intensidade e em seguida decrescem até o sétimo dia de cura, sendo que este processo ocorre mais rapidamente com a redução do teor de CAC. Dois aspectos são os principais responsáveis por este comportamento: 1) uma quantidade maior de brucita é formada (Fig. 1d), o que implica em maiores níveis de tensão e deformação da estrutura; 2) o teor de água utilizado no processamento dos concretos contendo MC2 é maior (Tabela III), o que ocasiona uma redução na resistência mecânica [20]. Além disto, a redução do teor de CAC acarreta normalmente uma diminuição adicional da resistência mecânica, resultando na formação de trincas maiores, como verificado por meio da comparação do aspecto visual dos concretos MC26CAC e MC2-4CAC com a composição MC2-2CAC (Fig. 4), e num aumento mais acentuado da porosidade (Fig. 3). Estes resultados indicam que a seleção do teor de CAC deve também levar em consideração a reatividade da fonte de magnésia utilizada.

Em relação aos concretos contendo cimento aluminoso, os concretos ligados por magnésia apresentam menores valores de MOE (Fig. 2) e porosidade semelhante ou inferior (Fig. 3). No entanto, na ausência de cimento, a porosidade da estrutura permite a acomodação de uma quantidade maior de brucita (Fig. 1) sem os problemas de expansão associados, como observado para as composições SM-0CAC e MC10CAC. Além disto, mesmo quando ocorre a formação de trincas (concreto MC2-0CAC, Fig. 2d), o aspecto visual dos concretos indica que na ausência de CAC o dano é muito menor (Fig. 4).
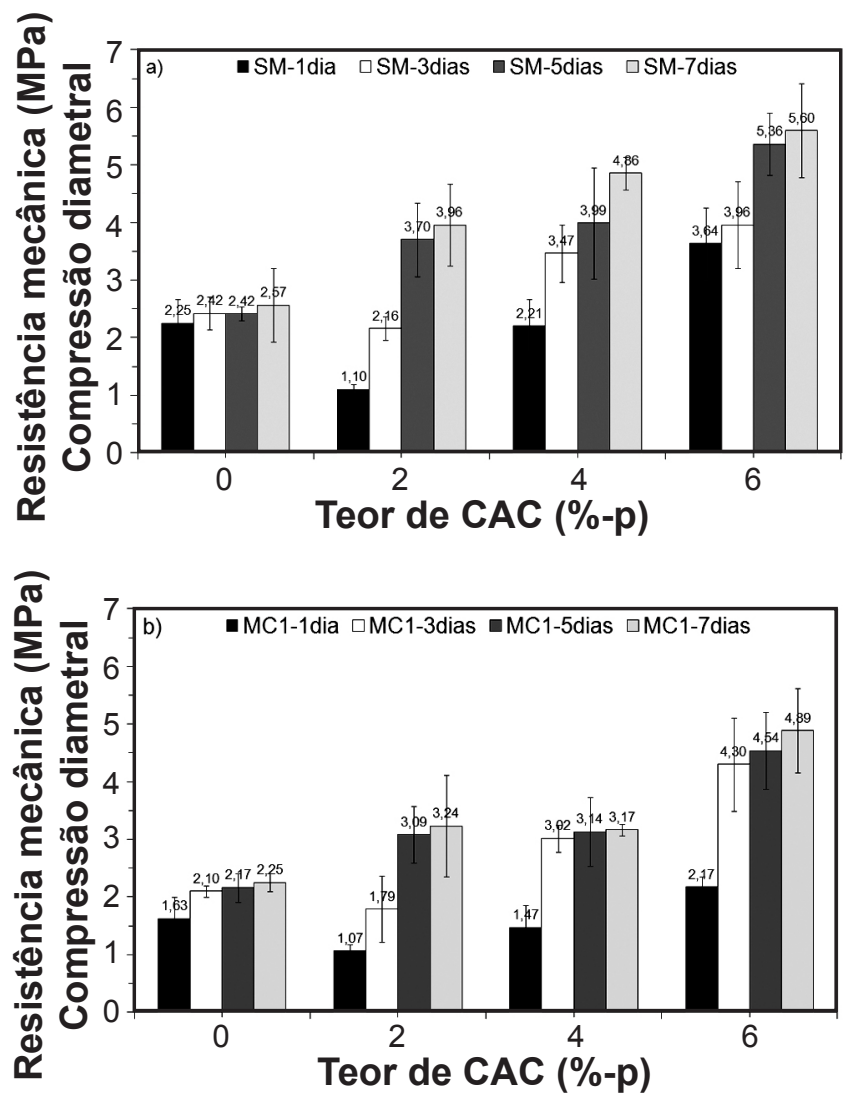

Figura 5: Resistência mecânica (compressão diametral) em função do tempo de cura para os concretos contendo diferentes teores de cimento (6CAC, 4CAC, 2CAC ou 0CAC) e fontes $\mathrm{MgO}$ : a) contendo $\mathrm{SM}$ e b) contendo $\mathrm{MC} 1$.

[Figure 5: Mechanical strength (diametral compression) as a function of the curing time for castables with different cement contents (6CAC, 4CAC, 2CAC or 0CAC) and $\mathrm{MgO}$ sources: a) containing SM and b) containing MC1.]

Com o intuito de comparar o desempenho dos concretos que não apresentaram danos (composições contendo SM e $\mathrm{MC1}$ ) com o valor mínimo de resistência mecânica à verde necessário para uma desmoldagem adequada (> $1 \mathrm{MPa}$ de compressão diametral [21]), realizou-se ensaios de resistência mecânica cujos resultados são apresentados na Fig. 5. Com o aumento do teor de CAC observa-se um aumento da resistência mecânica tanto para os concretos contendo SM (Fig. 5a) quanto para as composições contendo MC1(Fig. 5b), sendo que todas as composições atingem a resistência mínima requerida pela indústria. A resistência mecânica dos concretos SM-0CAC e MC1-0CAC após 1 dia de cura é equivalente às composições semelhantes contendo 4\%-p de CAC, indicando o potencial da magnésia como ligante para estes materiais. Entretanto, para os concretos ligados por cimento observa-se um aumento mais pronunciado da resistência mecânica em função do tempo de cura, o que está relacionado às diferenças entre os mecanismos de hidratação do $\mathrm{CAC}$ e do $\mathrm{MgO}$.

\section{Avaliação da etapa de secagem e tratamento térmico}

Após a cura, os concretos possuem maior rigidez e sua 

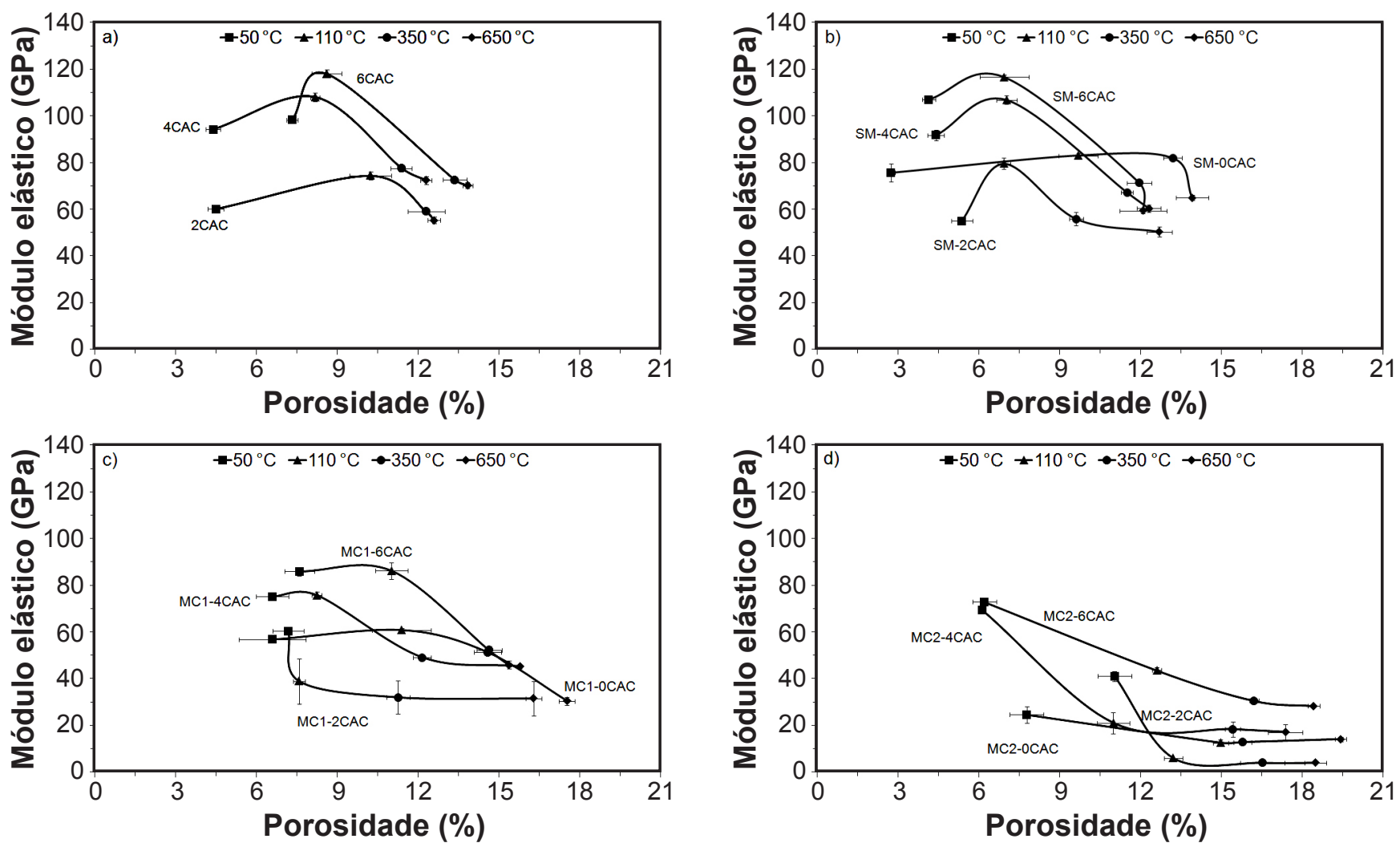

Figura 6: Modulo elástico em função da porosidade aparente para concretos contendo diferentes teores de cimento (6CAC, 4CAC, 2CAC ou $0 \mathrm{CAC}$ ), com ou sem $\mathrm{MgO}$ após 1 dia de cura e após secagem a $110^{\circ} \mathrm{C}, 350{ }^{\circ} \mathrm{C}$ e $650{ }^{\circ} \mathrm{C}$ : a) sem $\mathrm{MgO}$; b) contendo $\mathrm{SM}$; c) contendo $\mathrm{MC} 1$ e d) contendo MC2.

[Figure 6: Elastic modulus as a function of the apparent porosity for castables with different cement contents (6CAC, 4CAC, 2CAC or $O C A C$ ) with or without $\mathrm{MgO}$ after 1 day of curing and drying at $110^{\circ} \mathrm{C}, 350^{\circ} \mathrm{C}$ and $650{ }^{\circ} \mathrm{C}$ : a) $\mathrm{MgO}$-free; b) containing SM; c) containing $M C 1$ and d) containing MC2.]

capacidade de acomodar adequadamente a expansão gerada pela formação de brucita é reduzida e por isto estão mais sujeitos ao trincamento. Além disto, como a velocidade de hidratação da magnésia aumenta consideravelmente com a temperatura [22, 23] e também é acelerada devido à reação com o vapor de água [24], a avaliação da integridade física dos concretos refratários após a secagem e tratamento térmico torna-se ainda mais relevante. Souza e co-autores [18] indicam que os efeitos da hidratação do $\mathrm{MgO}$ nesta etapa do processamento podem ser avaliados com precisão por meio de medidas de MOE.

Os resultados do módulo elástico e da porosidade para os concretos produzidos após 1 dia de cura a $50^{\circ} \mathrm{C}$ e tratados termicamente em diferentes temperaturas $\left(110{ }^{\circ} \mathrm{C}, 350{ }^{\circ} \mathrm{C}\right.$ e $650{ }^{\circ} \mathrm{C}$ ) são apresentados na Fig. 6 . De forma geral, para concretos contendo cimento aluminoso e sem $\mathrm{MgO}$, entre $50{ }^{\circ} \mathrm{C}$ e $110{ }^{\circ} \mathrm{C}$, espera-se simultaneamente um aumento da porosidade e do MOE resultante da saída da água livre, da aproximação das partículas e da conversão de hidratos metaestáveis do cimento aluminoso, $\mathrm{C}_{2} \mathrm{AH}_{8}$ e $\mathrm{CAH}_{10}$, para o hidrato estável $\mathrm{C}_{3} \mathrm{AH}_{6}[12]$. Em temperaturas mais elevadas, entre $110{ }^{\circ} \mathrm{C}$ e $650{ }^{\circ} \mathrm{C}$, a decomposição dos hidratos $\mathrm{C}_{2} \mathrm{AH}_{8}$ $\left(2 \mathrm{CaO} \cdot \mathrm{Al}_{2} \mathrm{O}_{3} \cdot 8 \mathrm{H}_{2} \mathrm{O}\right), \mathrm{AH}_{3}\left(\mathrm{Al}_{2} \mathrm{O}_{3} \cdot 3 \mathrm{H}_{2} \mathrm{O}\right)$ e $\mathrm{C}_{3} \mathrm{AH}_{6}(3 \mathrm{CaO}$. $\mathrm{Al}_{2} \mathrm{O}_{3} \cdot 6 \mathrm{H}_{2} \mathrm{O}$ ), como indicado anteriormente (Fig. 1a), é acompanhada por um aumento da porosidade e uma redução do MOE. Este comportamento pode ser observado para os concretos 6CAC, 4CAC e 2CAC, como indicado na Fig. 6a.

Quando o $\mathrm{MgO}$ é adicionado, algumas diferenças no MOE dos concretos em função da temperatura podem ser observadas dependendo da formação de trincas ou não. Entre $50{ }^{\circ} \mathrm{C}$ e $110{ }^{\circ} \mathrm{C}$, a hidratação do $\mathrm{MgO}$ contribui para o aumento do MOE, quando a expansão é devidamente acomodada na estrutura. Por outro lado, uma redução do MOE neste mesmo intervalo de temperatura é uma indicação que a hidratação do $\mathrm{MgO}$ gerou trincas nos concretos [18]. Entre $110^{\circ} \mathrm{C}$ e $350^{\circ} \mathrm{C}$, uma redução significativa do MOE elástico é esperada para os concretos contendo cimento de aluminato de cálcio, devido à decomposição de seus hidratos [12], enquanto para os concretos sem cimento ocorre apenas a eliminação da água livre remanescente [18]. Finalmente, entre $350{ }^{\circ} \mathrm{C}$ e $650{ }^{\circ} \mathrm{C}$, uma diminuição adicional do MOE e um aumento da porosidade são esperados devido à decomposição da brucita.

O comportamento dos concretos contendo CAC e a magnésia menos reativa (SM) foi similar ao observado para as composições sem $\mathrm{MgO}$, indicando que a hidratação da magnésia durante os tratamentos térmicos não gerou danos, já que é baixa a formação de brucita (Fig. 6a e b). No caso dos concretos contendo $\mathrm{MC} 1$, entre $50^{\circ} \mathrm{C}$ e $110^{\circ} \mathrm{C}$, o aumento do MOE para as composições MC1-6CAC e MC1- 
4CAC é bem pequeno enquanto para o concreto MC1-2CAC observa-se uma redução do MOE no mesmo intervalo de temperatura indicando o trincamento do concreto (Fig. 6c). Para as composições contendo MC2 (Fig. 6d), a expansão associada a hidratação do $\mathrm{MgO}$ é ainda maior, resultando no trincamento de todos os concretos, como indicado pela redução do MOE entre $50{ }^{\circ} \mathrm{C}$ e $110{ }^{\circ} \mathrm{C}$. Estes resultados enfatizam a importância da análise da integridade física dos concretos durante a cura e a secagem, destacando novamente que a seleção do teor de CAC deve considerar a reatividade da fonte de $\mathrm{MgO}$ utilizada. Em comparação com os concretos contendo CAC, os concretos ligados por magnésia apresentam um aumento significativamente maior da porosidade entre $50{ }^{\circ} \mathrm{C}$ e $110{ }^{\circ} \mathrm{C}$ (Fig. 6 b-d), conseqüentemente, uma quantidade maior de brucita pode ser acomodada de forma que os danos são evitados (SM0CAC, MC1-0CAC) ou, pelo menos, reduzidos (MC20CAC). Após o tratamento térmico a $650{ }^{\circ} \mathrm{C}$ já houve a decomposição de todas as fases hidratadas formadas durante a mistura e cura dos concretos. Para tratamentos térmicos em temperaturas superiores, os concretos só apresentarão mudanças significativas de suas propriedades a partir do início da formação das ligações cerâmicas, onde é observado um aumento do MOE e também da resistência mecânica, uma vez que estas propriedades estão correlacionadas. Desta forma, pode-se afirmar que para as composições SM-0CAC e MC1-0CAC seria dispensado o uso de cimento, reduzindo os custos do concreto e ao mesmo tempo evitando a formação de fases de indesejáveis durante seu uso.

\section{CONCLUSÕES}

Neste estudo são apresentadas rotas para a incorporação de magnésias com diferentes reatividades aos concretos refratários, sem os danos decorrentes da expansão volumétrica associados à hidratação do $\mathrm{MgO}$. Para magnésia com baixa reatividade (SM), a hidratação do $\mathrm{MgO}$ é facilmente controlada e os concretos apresentam excelente desempenho. Uma quantidade maior de brucita é formada para composições contendo a magnésia com reatividade média-alta ( $\mathrm{MC1}$ ), mas não são observados efeitos deletérios para os concretos MC1-6CAC e MC14CAC, indicando que por meio da utilização desta fonte de magnésia é possível explorar os benefícios da redução da temperatura de início de formação de espinélio. O concreto MC1-2CAC não apresentou trincas durante a cura, mas uma redução do MOE foi observada após a secagem a $110{ }^{\circ} \mathrm{C}$, enfatizando a importância da avaliação da integridade mecânica dos concretos após a secagem quando o objetivo é a incorporação de magnésia mais reativa. Tal comportamento indica que para os concretos contendo CAC e $\mathrm{MgO}$, o teor mínimo de cimento para garantir que os danos associados à hidratação sejam evitados é mais elevado para fontes de magnésia cáustica, destacando que reatividade da magnésia deve ser considerada na escolha do teor de CAC. A adição de até $6 \%$-p de cimento é suficiente para evitar os efeitos deletérios da hidratação quando são utilizadas as magnésias $\mathrm{SM}$ e $\mathrm{MC1}$, mas o mesmo não foi observado para a fonte MC2. Neste caso, teores mais elevados de CAC ou a adição de outros aditivos anti-hidratação precisam ser avaliados. Os concretos SM-0CAC e MC1-0CAC apresentaram resistência mecânica após 1 dia de cura equivalentes aos valores observados para composições semelhantes contendo 4\%-p de CAC e, após o tratamento térmico à $650{ }^{\circ} \mathrm{C}$, o módulo elástico e a avaliação visual das amostras confirmaram a ausência de trincas. Sendo assim, como o MOE está correlacionado com a resistência mecânica, este comportamento indica que o $\mathrm{MgO}$ pode ser utilizado para a substituição do cimento de aluminato de cálcio, possibilitando uma redução significativa nos custos do concreto refratário além de evitar à formação de fases de baixa refratariedade.

\section{AGRADECIMENTOS}

Os autores agradecem à Magnesita Refratários S.A., FAPESP e CNPq pelo apoio fornecido ao trabalho.

\section{REFERÊNCIAS}

[1] M. A. Braulio, D. H. Milanez, E. Y. Sako, L. R. M. Bittencourt, V. C. Pandolfelli, "Are refractory agregates inert?”, Am. Ceram. Soc. Bull. 87, 3 (2007) 27-32.

[2] M. A. Braulio, L. R. M. Bittencourt, J. Poirier, V. C. Pandolfelli, "Microssilica effects on cement bonded alumina-magnesia refractory castables", J. Tech. Assoc. Refract. Jnp. 28, 3 (2008) 180-184.

[3] M. A. Braulio, L. R. M. Bittencourt, V. C. Pandolfelli, "Magnesia grain size effect on in situ spinel refractory castables", J. Eur. Ceram. Soc. 28 (2008) 2845-2852.

[4] H. S. Tripathi, B. Mukherjee, S. Das, M. K. Haldar, S. K. Das, A. Ghosh, "Synthesis and densification of magnesium aluminate spinel: effect of $\mathrm{MgO}$ reactivity", Ceram. Inter. 29 (2003) 915-918.

[5] M. A. Braulio, J. F. R. Castro, C. Pagliosa, L. R. M. Bittencourt, V. C. Pandolfelli, "From macro to nano magnesia: designing the in situ spinel expansion", J. Am. Ceram. Soc. 91, 9 (2008) 3090-3093.

[6] R. Salomão, L. F. Amaral, V. C. Pandolfelli, "Adição de cimento de aluminato de cálcio e seus efeitos na hidratação do óxido de magnésio", Cerâmica 56, 338 (2010) 135-140.

[7] R. Salomão, V. C. Pandolfelli, "The role of hydraulic binders on magnesia containing refractory castables: calcium aluminate cement and hydratable alumina”, Ceram. Inter. 35 (2009) 3117-3124.

[8] R. Salomão, V. C. Pandolfelli, "Efeito da adição de microssílica na hidratação do óxido de magnésio em concretos refratários", Cerâmica 54 (2008) 43-48.

[9] M. A. Braulio, D. H. Milanez, E. Y. Sako, L. R. M. Bittencourt, V. C. Pandolfelli, "Expansion behaviour of cement-bonded alumina-magnesia refractory castables", Am. Ceram. Soc. Bull. 86, 12 (2007) 9201-9206.

[10] B. Sandberg, T. Mosberg, "Use of microsilica in binder systems for ultra-low cement castables and basic, cement- 
free castables", Ceramic Transaction 4 (1989) 245-258.

[11] B. Myrhe, B. Sandberg, "The use of microsilica in refractory castables", disponível em: www.refractories. elkem.com/dav/b360ef3589.PDF, acesso Janeiro (2011).

[12] W. E. Lee, W. Vieira, S. Zhang, K. Ghanbari Ahari, H. Sarpoolaky, C. Parr, "Castable refractory concretes", Inter. Mat. Ver. 46, 3 (2001) 145-167.

[13] G. Pickett, "Equations for computing elastic constants from flexural and torsional resonant frequencies of vibration of prisms and cylinders", Proc. Am. Soc. Testing Mater. 45 (1945) 846-865.

[14] M. D. M. Innocentini, F. A. Cardoso, M. M. Akyioshi, V. C. Pandolfelli, "Drying stages during the heating of highalumina, ultra-low-cement refractory castables", J. Am. Ceram. Soc. 86, 7 (2003) 1146-48.

[15] T. M. Souza, M. A. L Braulio, P. Bonadia, V. C. Pandolfelli, "Hidratação da magnésia e seu efeito ligante em concretos refratários sem cimento", Cerâmica 59, 350 (2010) 2013-215.

[16] R. Salomão, L. R. Bittencourt, V. C. Pandolfelli, "A novel approach for magnesia hydration assessment in refractory castables", Ceram. Inter. 33 (2007) 803-810.

[17] R. Salomão, V.C. Pandolfelli, "Hidratação e desidratação de óxido de magnésio em concretos refratários", Cerâmica 54, 330 (2008) 145-151.
[18] T. M. Souza, M. A. L. Braulio, P. Bonadia, V. C. Pandolfelli, "Módulo elástico como ferramenta para avaliação da hidratação da magnésia e do processamento de concretos refratários", Cerâmica 58, 347 (2012) 301-312.

[19] J. R. Garcia, I. R. de Oliveira, V. C. Pandolfelli, "Processo de hidratação e os mecanismos de atuação dos aditivos aceleradores e retardadores de pega do cimento de aluminato de cálcio", Cerâmica 53, 325 (2007) 42-56.

[20] F. T. Ramal Jr., R. Salomão, V. C. Pandolfelli, "Water content and its effect on the drying behaviour of refractory castabes", Ref. Appl. News 10, 3 (2005) 10-13.

[21] C. Parr, L. Bin, B. Valdelièvre, C. Wöhrmeyer, B. Touzo, "The advantages of calcium aluminate cement containing castables for steel ladle applications", Proc. XXXII ALAFAR, Antigua, Guatemala (2004).

[22] A. Kitamura, K. Onizuka, K. Tanaka, "Hydration characteristics of magnesia", Taikabutsu Overseas 16, 3 (1995) 3-11.

[23] S. D. F Rocha, M. B. Mansur, V. S. T. Ciminelli, "Kinetics and mechanalistic analysis of caustic magnesia hydration", J. Chem. Technol. Biotechnol. 79, 8 (2004) 816821.

[24] G. K. Layden, G. W. Brindley, "Kinetics of vaporphase hydration of magnesium oxide", J. Am. Ceram. Soc. 46, 11, (1963) 518-522.

(Rec. 09/02/2011, Ac. 10/06/2011) 